\title{
Teleasistencia psicológica para niños con Trastorno del Espectro Autista: definiciones, directrices y tendencias tecnológicas para el trabajo basado en evidencia
}

\author{
Connie Aliaga Guanilo ${ }^{2}$, Tommy Lopez-Fernández ${ }^{12}$, \\ Astry Torres-Chamorro ${ }^{12}$ y Betsabe Vasquez-Florentino ${ }^{12}$ \\ ${ }^{1}$ Instituto Psicopedagógico EOS Perú \\ 2Universidad Nacional Mayor de San Marcos
}

\begin{abstract}
Resumen
En respuesta a la situación actual causada por la pandemia de COVID-19, se describe la teleasistencia psicológica como alternativa de trabajo, la cual permita a los profesionales del área de psicología y de lenguaje, internistas, padres de familia y/o cuidadores de los niños que asisten al programa Mundo TEA, continuar otorgando y recibiendo un servicio psicológico de calidad. Este documento aborda los deslindes conceptuales de la teleasistencia psicológica, analizando las ventajas y desventajas de este servicio; así como las estrategias y técnicas del Análisis Conductual Aplicado para garantizar una atención basada en evidencia científica. Así mismo, se precisan los tipos de recursos tecnológicos e instrumentos requeridos en el proceso de evaluación e intervención psicológica de forma remota. Por otro lado, se detallan directrices de las funciones y roles para los participantes involucrados en este sistema de asistencia, bajo los aspectos teóricos, prácticos y éticos respectivos. Finalmente, se construye un modelo de consentimiento informado, con el fin de garantizar al usuario una atención de la forma más seria, confidencial y profesional posible.
\end{abstract}

Palabras clave: análisis conductual aplicado, intervenciones basadas en evidencia, recursos tecnológicos, teleasistencia psicológica, trastorno del espectro autista.

\section{Introducción}

\section{Trastorno del Espectro Autista (TEA)}

El trastorno del Espectro Autista (TEA) hace referencia a un conjunto de alteraciones del neurodesarrollo que se caracterizan, principalmente, por deficiencias en la interacción y comunicación social, así como por intereses y conductas restringidas $y$ repetitivas (American Psychiatric Association, 2013; Alcantud et al., 2017; Ministerio de la mujer y poblaciones vulnerables, 2017; Organización Mundial de la Salud, 2019; Will et al., 2018). Dichas características se muestran de manera heterogénea dependiendo del individuo y etapa del ciclo vital.

La evidencia actual demuestra que intervenciones tempranas, entre los 02 y 05 años, son altamente significativas, ya que aumentan la posibilidad de disminuir la severidad de la sintomatología en los niños con TEA (Rojas et al., 2019; Will et al., 2018). En específico, las intervenciones basadas en los principios del Análisis Conductual Aplicado (ACA) son muy eficaces (Sandbank et al., 2020). Sin embargo, por factores económicos, ambientales y/o situacionales, se podría presentar dificultades para acceder o mantener dicha intervención de forma presencial. Por ejemplo, actualmente, la situación de pandemia por COVID-19 ha provocado que la mayor parte de gobiernos tomen medidas de control, como el aislamiento social o cuarentena, lo cual está imposibilitando asistir a las terapias tradicionales (Brooks et al., 2020). Por lo que, se debe recurrir a alternativas de trabajo como es la teleasistencia psicológica, pues esta respondería a las necesidades de abordar las áreas en déficit de los niños con TEA mediante el asesoramiento y entrenamiento a padres (Pennefather et al., 2018).

\section{Teleasistencia psicológica}

La teleasistencia psicológica se aplica mediante diferentes tecnologías que permiten una comunicación no presencial, siendo esta sincrónica (la transmisión se da en tiempo real) - asincrónica (el paciente transmite la información y el profesional la revisa sin la presencia del primero) (Boisvert et al., 2010), y buscando proporcionar atención psicológica a personas que residen en un lugar de diferente ubicación física que el especialista, y en situaciones en las que no es posible 
desplazarse hacia el lugar asistencial (American Psychological Association, 2013; Colegio Colombiano de Psicólogos, 2020). Por otro lado, si bien, existen diversos conceptos involucrados (Tabla 1), se utilizará a lo largo del documento, el término de teleasistencia psicológica pues se adecúa a las características y fines del trabajo, así como al contexto de pandemia por COVID-19. Aunque esta modalidad no presencial de prestación de servicios presenta diferentes beneficios, se han encontrado también algunas limitaciones en su aplicación (Tabla 2).

El presente trabajo busca informar sobre el funcionamiento y procedimiento de la teleasistencia psicológica, describir los roles y funciones de los involucrados e indicar las estrategias, técnicas, instrumentos y recursos tecnológicos existentes, que permitan una atención online de calidad y basada en evidencia científica.

Tabla 1

Conceptos relacionados a la teleasistencia psicológica

\begin{tabular}{cc}
\hline Concepto & Definición \\
\hline Teleasistencia $^{3}$ & Realización de sesiones de rehabilitación logopédica mediante \\
plataformas virtuales.
\end{tabular}

Tabla 2

Beneficios y limitaciones de la teleasistencia psicológica

\begin{tabular}{ccc}
\hline Criterio & Beneficios & Limitaciones \\
\hline & $\begin{array}{c}\text { Incluye interacciones sincrónicas y } \\
\text { almacenamiento asíncrono. }\end{array}$ & $\begin{array}{c}\text { Relacionadas a la estabilidad de la } \\
\text { conexión de internet o al aparato. }\end{array}$ \\
$\begin{array}{c}\text { Aspectos } \\
\text { técnicos }\end{array}$ & $\begin{array}{c}\text { Mejora la continuidad de la atención al } \\
\text { paciente. }\end{array}$ & $\begin{array}{c}\text { No es posible utilizar ayudas físicas o el } \\
\text { modelado en vivo, por lo que se sirve de } \\
\end{array}$ \\
& $\begin{array}{c}\text { Supera las barreras geográficas o } \\
\text { temporales. }\end{array}$ & las guías verbales. \\
\hline
\end{tabular}

\begin{tabular}{ccc}
\hline $\begin{array}{c}\text { Aspectos } \\
\text { éticos }\end{array}$ & $\begin{array}{c}\text { Se crean límites y expectativas de la } \\
\text { relación formal mediante el consentimiento } \\
\text { informado. } \\
\text { Se permite la flexibilidad a medida que } \\
\text { evoluciona la práctica de teleasistencia. }\end{array}$ & $\begin{array}{c}\text { Problemas relacionados a la privacidad, } \\
\text { identificación y confidencialidad de los } \\
\text { datos. }\end{array}$ \\
\hline $\begin{array}{c}\text { Aspectos } \\
\text { del } \\
\text { emisor }{ }^{1}\end{array}$ & Flexibilidad, ahorro de tiempo y dinero. & $\begin{array}{c}\text { Problemas de la capacitación de los } \\
\text { profesionales. }\end{array}$ \\
Mejora el uso del tiempo. & $\begin{array}{c}\text { Resistencia para usar recursos } \\
\text { tecnológicos. }\end{array}$
\end{tabular}


Mejora y facilita la supervisión del tratamiento.

Aspectos

del receptor $^{2}$
Reduce el costo y tiempo de traslado.

Beneficia a pacientes con dificultades de desplazamiento (situación nacional).
Impacto negativo en la relación del profesional y padres.

Frustración o reducción del interés por parte de los participantes.

Manejo de recursos tecnológicos por ciertos grupos de pacientes.

Falta de interacciones sociales directas.

Nota. Esta tabla muestra los principales beneficios y limitaciones de la teleasistencia psicológica (Camargo \& Marino, 2020; McCord et al., 2020; Mougela, 2019; Sulkowski, 2015; Unholz-Bowden et al., 2020 y Zhu, 2019).

${ }^{1}$ Psicólogo/ especialista/ internista. ${ }^{2}$ Padre o madre/ cuidador

\section{Consideraciones para la teleasistencia psicológica basada en evidencia científica}

\section{Público objetivo}

Dirigido a psicólogos, especialistas de lenguaje, internistas, padres de familia y cuidadores de niños pertenecientes al programa Mundo TEA, el cual involucra un trabajo sistematizado basado en el Análisis Conductual Aplicado para la evaluación e intervención psicológica en niños de 02 a 05 años con Trastorno del Espectro Autista.

\section{Estrategias y técnicas}

Es bien sabido que, se deben utilizar las mejores estrategias y técnicas basadas en evidencia científica para la realización de una adecuada evaluación e intervención psicológica (Sandbank et al., 2020). De tal manera que, lo mismo debe suceder cuando estas (evaluaciones e intervenciones) se dan de forma remota u online, ya que solo así se garantizará una atención psicológica de calidad.

A partir de lo mencionado anteriormente, se ha encontrado estudios, los cuales refieren que muchas de las estrategias y técnicas basadas en el Análisis Conductual Aplicado son las más adecuadas y respaldadas para ser usadas en el sistema de teleasistencia psicológica de niños con TEA, incluyendo las áreas de conducta, aprendizaje y de lenguaje (Douglas et al., 2017; Parsons, 2019; Pennefather et al., 2018; Sandbank et al., 2020; Unholz-Bowden et al., 2020; Vismara et al., 2013). Estos mismos estudios resaltan al análisis funcional de la conducta y la observación conductual como parte de las estrategias y técnicas más utilizadas y eficaces (Tabla 3) para la evaluación no presencial de niños pequeños.

Por otro lado, en relación con el trabajo de intervención, al ser niños no mayores de 05 años, esta deberá ser realizada directamente con los padres y/o cuidadores por medio del asesoramiento y entrenamiento (Larroy et al., 2020), puesto a que, estos serán quienes apliquen las estrategias y técnicas (Tabla 2) proporcionadas por el especialista según los requerimientos de cada caso (Pennefather et al., 2018). Por consiguiente, todo el contenido del entrenamiento a padres tendrá que estar enfocado en los momentos de aprendizaje dentro de las interacciones y actividades diarias que conforman la vida del niño pequeño (Vismara et al., 2013).

\section{Instrumentos y recursos tecnológicos}

Con respecto al uso de instrumentos (Tabla 4) para la evaluación de forma remota, es importante que estos mantengan la integridad de sus propiedades (fiabilidad y validez), de tal forma que, se puedan conservar las condiciones de administración indicadas en la prueba manual, sin olvidar considerar aspectos básicos como: calidad de las tecnologías a utilizar, viabilidad para que el sujeto pueda responder al instrumento de forma online $y$ se descarte posibles distracciones que puedan afectar al resultado (Ramos, 2017). Es así como, no todos los instrumentos podrán ser adaptados a una modalidad online, por lo que, se recomienda que la evaluación, en el caso de los niños con TEA, se base primordialmente, en el uso de listas de cotejos, la entrevista psicológica y el análisis funcional de la conducta.

En cuanto a los recursos tecnológicos (Tabla 4 ), es relevante mencionar que existen dos tipos: los tangibles (computadora, laptop, tablet o celular) y los intangibles (aplicativos, sistemas o programas) (Caicedo, 2015). En el caso del primer tipo, se debe garantizar que estos cuenten con conexión a internet, sistemas de audio y video; mientras que los del segundo tipo, deben tener un sistema de 
seguridad que permita asegurar la confidencialidad y privacidad de la teleasistencia psicológica, así como certeza que tanto el profesional como el paciente sepan utilizarlos (Colegio Logopedas de Murcia, 2020).

\section{Directrices}

Luego de la revisión de las estrategias y técnicas, así como los requisitos para tener en cuenta para en cuanto al uso de instrumentos y recursos tecnológicos; es necesario contar con un protocolo (Tabla 5) donde se describa el rol y funciones de cada participante en la teleasistencia psicológica.

\section{Tabla 3}

Estrategias y técnicas para la teleasistencia psicológica

\begin{tabular}{|c|c|c|c|c|}
\hline & \multicolumn{2}{|c|}{ Estrategias } & Técnicas & Medio $^{1}$ \\
\hline \multirow{2}{*}{$\begin{array}{l}\text { Evaluación } \\
\text { psicológica } \\
\text { de forma } \\
\text { remota }\end{array}$} & \multirow{2}{*}{\multicolumn{2}{|c|}{ Análisis Funcional de la Conducta }} & Observación & $\begin{array}{l}\text { En vivo por medio } \\
\text { de la plataforma }\end{array}$ \\
\hline & & & $\begin{array}{l}\text { conductual participante } \\
\text { y no participante }\end{array}$ & $\begin{array}{l}\text { Por videos } \\
\text { caseros enviado } \\
\text { por los padres }\end{array}$ \\
\hline \multirow{4}{*}{$\begin{array}{l}\text { Intervención } \\
\text { psicológica } \\
\text { de forma } \\
\text { remota }\end{array}$} & \multirow{4}{*}{$\begin{array}{l}\text { Psicoeducación } \\
\text { (asesoramiento y } \\
\text { entrenamiento) }\end{array}$} & $\begin{array}{l}\text { Control de } \\
\text { estímulos }\end{array}$ & \multirow{4}{*}{$\begin{array}{c}\text { Reforzamiento positivo } \\
\text { Reforzamiento negativo } \\
\text { Videomodelado } \\
\text { Extinción } \\
\text { Encadenamiento } \\
\text { Moldeamiento } \\
\text { Apoyos visuales }\end{array}$} & \multirow{2}{*}{$\begin{array}{c}\text { Rol activo del } \\
\text { padre y/o cuidador } \\
\text { del menor }\end{array}$} \\
\hline & & Estrategias & & \\
\hline & & antecedent & & \multirow[b]{2}{*}{$\begin{array}{l}\text { Especialista del } \\
\text { área }\end{array}$} \\
\hline & & $\begin{array}{c}\text { Estrategias } \\
\text { consecuentes }\end{array}$ & & \\
\hline
\end{tabular}

Nota. Esta tabla muestra las principales estrategias y técnicas con evidencia científica para el sistema de teleasistencia (Douglas et al., 2017; Larroy, 2020; Pennefather et al., 2018; Sandbank et al., 2020; Vismara et al., 2013). ${ }^{1}$ Medio por el cual se podrá ejecutar las técnicas y estrategias.

Tabla 4

Instrumentos y recursos tecnológicos para la teleasistencia psicológica

\begin{tabular}{|c|c|c|c|}
\hline & Tipos & Requisitos & Ejemplos \\
\hline \multirow[t]{3}{*}{ Instrumentos } & $\begin{array}{c}\text { Pruebas online } \\
\text { o adaptadas al } \\
\text { medio }\end{array}$ & Integridad de la fiabilidad y validez & $\begin{array}{c}\text { CARS } \\
\text { M-CHAT }^{1} \\
\text { CHAT }^{1} \\
\text { SCQ }^{1} \\
\text { ASA-HiCh }^{1}\end{array}$ \\
\hline & $\begin{array}{l}\text { Entrevista } \\
\text { psicológica }\end{array}$ & Calidad de las tecnologías & $\begin{array}{c}\text { Basadas en los } \\
\text { indicadores de TEA }\end{array}$ \\
\hline & $\begin{array}{l}\text { Listas de } \\
\text { cotejos }\end{array}$ & $\begin{array}{c}\text { Viabilidad que el sujeto responda sin } \\
\text { distracciones }\end{array}$ & EDLO \\
\hline & Tangibles & $\begin{array}{c}\text { Acceso a internet } \\
\text { Sistema de audio y video }\end{array}$ & $\begin{array}{c}\text { Computadora }{ }^{2} \\
\text { Laptop }^{2} \\
\text { Tablet } \\
\text { Celular }\end{array}$ \\
\hline
\end{tabular}

\section{Seguro}

Recursos tecnológicos
Compatible mínimo con Windows, Linux o macOS

Capacidad de mínimo de 10 participantes

Transferencia de archivos

Mensajería instantánea

Versión en español

Nota. Esta tabla muestra ejemplos de instrumentos y recursos tecnológicos que garantizan un adecuado sistema de teleasistencia (Colegio Logopedas de Murcia, 2015; Ramos, 2017). ${ }^{1}$ Instrumentos de cribado en 
español con mayor eficacia (Alonso-Esteban et al., 2020). ${ }^{2}$ Recomendable. ${ }^{3}$ Seguro. ${ }^{4}$ Medianamente seguro.

${ }^{5}$ Altamente seguro.

\section{Tabla 5}

Roles y funciones para los participantes de la teleasistencia psicológica

\begin{tabular}{|c|c|c|}
\hline Participante & Rol & Funciones \\
\hline $\begin{array}{l}\text { Psicólogo/ } \\
\text { especialista/ } \\
\text { internista }\end{array}$ & Emisor & $\begin{array}{l}\text { - Certificar que el receptor comprenda el proceso de } \\
\text { intervención. } \\
\text { - Manejar competentemente las condiciones tecnológicas y del } \\
\text { entorno en la intervención. } \\
\text { - Adaptar el uso de instrumentos y técnicas de intervención. } \\
\text { - Garantizar la confidencialidad de los receptores en base a las } \\
\text { normas legales, éticas y deontológicas. } \\
\text { - Remitir los informes de evaluación y reportes del proceso de } \\
\text { intervención. }\end{array}$ \\
\hline $\begin{array}{l}\text { Padre o madre/ } \\
\text { cuidador }\end{array}$ & $\begin{array}{l}\text { Receptor } \\
\text { directo }\end{array}$ & $\begin{array}{l}\text { - Conservar un espacio bajo condiciones óptimas para la } \\
\text { intervención. } \\
\text { - Manejar los recursos tecnológicos necesarios para la } \\
\text { teleasistencia. }\end{array}$ \\
\hline Niño/ a & $\begin{array}{l}\text { Receptor } \\
\text { indirecto }\end{array}$ & - Permanecer en el mismo espacio que el receptor directo. \\
\hline
\end{tabular}

Nota. Esta tabla muestra los roles y las principales funciones que cumplen los participantes en teleasistencia psicológica (American Psychological Association, 2013; De la Torre y Pardo, 2018; Larroy et al., 2020).

\section{Consideraciones éticas}

Es importante la capacitación adecuada, supervisión y/o consulta para el desarrollo de la práctica en teleasistencia psicológica, así como hacer énfasis en los aspectos éticos (American Psychological Association, 2013), con el fin de asegurar la protección de los datos personales y confidencialidad del paciente $e$ involucrados a través de un consentimiento informado (Anexo 1) donde se exponga la finalidad del uso de los datos que se le requiera.

\section{Conclusiones}

La teleasistencia psicológica es una alternativa de trabajo que, si bien ya existía, está tomando mayor fuerza este año, debido a la situación de cuarentena por el brote de COVID-19. Así mismo, es importante conocer tanto los procedimientos respaldados por la evidencia como las normas éticas que tiene esta modalidad de trabajo, pues solo así se podrá ofrecer servicios psicológicos de calidad a los diversos usuarios, siendo en este caso, los niños con Trastorno del Espectro Autista.

\section{Referencias}

Alcantud, F., Alonso, Y., \& Mata, S. (2017). Prevalencia de los trastornos del espectro autista: revisión de datos.
Universidad de Salamanca. 47(4), 726. http://dx.doi.org/10.14201/scero20164 74726

Alonso-Esteban, Y., Marco, R., Hedley, D., Uljarevié, M., Barbaro, J., Canal-Bedia, R., \& Alcantud-Marín, F. (2020). Screening instruments for early detection of autism spectrum disorder in Spanish speaking communities. Psicothema, 32(2), 245-252. https://doi.org/10.7334/psicothema201 9.340

American Psychiatric Association (2013). Manual diagnóstico y estadístico de los trastornos mentales (DSM-5). Médica Panamericana.

American Psychological Association (2013). Guidelines for the practice of Telepsychology. American Psychologist, $\quad$ 68(9), 791-800. https://doi.org/10.1037/a0035001

Boisvert, M., Russell, L., Andrianopoulos, M., \& Boscardin M. L. (2010). Telepractice in the assessment and treatment of individuals with autism spectrum disorders: A systematic review. Developmental Neurorehabilitation, 13(6),
423-432. 
https://doi.org/10.3109/17518423.2010 .499889

Brooks, S., Webster, R., Smith, L., Woodland, L., Wessely, S., Greenberg, N., \& James, G. (2020). The Psychological impact of quarantine and how to reduce it: rapid review of the evidence. The Lancet, 395(10227), 912-920. https://doi.org/10.1016/S01406736(20)30460-8

Caicedo, S. (2015). Los recursos tecnológicos educativos y su influencia en el rendimiento académico de los estudiantes de educación básica media de la escuela "Juan Francisco Montalvo" del Cantón Píllaro, provincia de Tungurahua [Tesis de grado, Universidad Técnica de Ambato]. https://cutt.ly/uyaaV4j

California Association for Behavior Analysis (23 de marzo de 2020). Telehealth and the practice of Applied Behavior Analysis (ABA). https://cutt.ly/0yaSaWh

Camargo, S., \& Marino, M. C. (2020). Teleconsultation: An Integrative Review of the Doctor-Patient interaction mediated by technology. Revista Brasileira de Educação Médica, $44(1)$. https://doi.org/10.1590/1981-

5271v44.1-20190224.ing

Colegio Colombiano de Psicólogos (21 de marzo de 2020). Comunicado a los profesionales de la Psicología sobre telepsicología. https://cutt.ly/3yaSpLx

Colegio Logopedas de Murcia (2020). Guía Teleasistencia Logopedia COVID-19. Consejo General de Colegios de Logopedas. https://cutt.ly/lyaaTE4

De la Torre, M., y Pardo, R. (2018). Guía para la Intervención Telepsicológica. Centro de Psicología Aplicada de la Universidad Autónoma de Madrid en colaboración con el Colegio Oficial de Psicólogos de Madrid. https://cutt.ly/fyaGwc0

Douglas, S., Kammes, R., \& Nordquist, E. (2017). Online Communication Training for Parents of Children with Autism Spectrum Disorder. Communication Disorders Quarterly, 39(3). https://doi.org/10.1177/152574011772 7491

Ferguson, J., Craig, E. A., \& Dounavi, K. (2018). Telehealth as a Model of providing Behaviour Analytic interventions to Individuals with Autism Spectrum Disorder: A Systematic Review. Journal of Autism and Developmental Disorders, 49, 582-616. https://doi.org/10.1007/s10803-0183724-5

Larroy, C., Estupiña, F., Fernández-Arias, I., Hervás, G., Valiente, C., Gómez, M., Crespo, M., Rojo, N., Roldán, L., Vázquez, C., Ayuela, D., Lozano, B., Martínez, A., Pousada, T., Gómez, A., Asenjo, M., Rodrigo, J., Florido, R., Vallejo, M., Sanz, S., ... Pardo, R. (2020). Guía para el abordaje no presencial de las consecuencias psicológicas del brote epidémico de COVID-19 en la población general. Universidad Complutense de Madrid. https://cutt.ly/4yaapra

McCord, C., Bernhard, P., Walsh, M., Rosner, C., \& Katie, C. (2020). A consolidated model for telepsychology practice. Journal of Clinical Paychology. https://doi.org/10.1002/jclp.22954

Ministerio de la mujer y poblaciones vulnerables (2017). Plan Nacional para las personas con Trastorno del Espectro Autista - TEA. Lima, Perú. https://cutt.ly/FyaSk6e

Mougela, V. (2019). Conception et implantation d'un dispositif de formation en ligne pour les parents d'enfants avec un trouble du spectre de l'autisme: adaptation en format à distance $d u$ programme psychoéducatif "'A.B.B.C. du comportement d'enfant ayant un TSA: des parents en action" [Thèse de doctorat, Université de Strasbourg]. https://cutt.ly/dyaSsQ7

Organización Mundial de la Salud (2019). Clasificación Internacional de Enfermedades (CIE-11). https://cutt.ly/jysqUR9

Parsons, D. N. (2019). Autism in regional and remote communities: Examining the effect of an autism spectrum disorder diagnosis on regional and remote families and innovative therapies [PhD Thesis, Curtin University] https://cutt.ly/dyaSde2

Pennefather, J., Hieneman, M., Raulston, T., \& Caraway, N. (2018). Evaluation of an online training program to improve family routines, parental well-being, 
and the behavior of children with autism. Research in Autism Spectrum Disorders, 21-26. https://doi.org/10.1016/j.rasd.2018.06. 006

Ramos, R. (ed.). (2017). Guía para la práctica de la telepsicología. Consejo Nacional de la Psicología España. https://doi.org/10.23923/cop.telepsicol ogia.2017

Rojas, V., Rivera, A., y Nilo, N. (2019). Actualización en diagnóstico e intervención temprana del Trastorno del Espectro Autista. Revista chilena de pediatría, 90(5), 478-484. https://doi.org/10.32641/rchped.v90i5. 1294

Sandbank, M., Bottema-Beutel, K., Crowley, S., Cassidy, M., Dunham, K., Feldman, J. I., Crank, J., Albarran, S. A., Raj, S., Mahbub, P., \& Woynaroski, T. G. (2020). Project AIM: Autism intervention meta-analysis for studies of young children. Psychological Bulletin, 146(1), 129. https://doi.org/10.1037/bul0000215

Shire, S. Y., Baker, L., Shih, W., \& Kasari, C. (2020). Comparison of Face-to-Face and Remote Support for Interventionist Learning to Delover JASPER Intervention with Children who have Autism. Journal of Behavioral Education.

https://doi.org/10.1007/s10864-02009376-4

Sulkowski, A. (2015). Comparison and staff preference on Teletheraphy versus in Person Therapy. Southern Illinois University Carbondale, Estados Unidos. https://cutt.ly/1yaSfmK

Unholz-Bowden, E., McComas, J. J., McMaster, K. L., Girtler, S. N., Kolb, R. L., \& Shipchandler, A. (2020). Caregiver training via telehealth on Behavioral Procedures: A systematic Review. Journal of Behavioral Education.

https://doi.org/10.1007/s10864-02009381-7

Vismara, L., McComick, C., Young, G., Nadhan, A., \& Monlux, K. (2013). Preliminary Findings of a Telehealth Approach to Parent Training in Autism. Journal of Autism and Developmental Disorders, 43, 2953-2969. https://doi.org/10.1007/s10803-0131841-8

Will, M., Currans, K., Smith, J., Weber, S., Duncan, A., Burton, J., KroegerGeoppinger, K., Miller, V., Stone, M., Mays, L., Luebreacht, A., Heeman, A., Erickson, C., \& Anixt, J. (2018). Evidenced-Based Interventions for Children with Autism Spectrum Disorder. Current Problems in Pediatric and Adolescent Health Care, 1237. https://doi.org/10.1016/j.cppeds.20 18.08.014

Zhu, J. (2019). A comparison of two types of remote performance feedback on treatment integrity [PhD Thesis, University of lowa]. https://doi.org/10.17077/etd.hpbi-n26t 
Anexo 1. Consentimiento Informado

\section{Anexos}

\section{CONSENTIMIENTO INFORMADO}

En el Instituto Psicopedagógico EOS Perú otorgamos mucha importancia a la información que nos brinda, por ello hemos implementado el servicio de teleasistencia psicológica para continuar prestando nuestra atención de la forma más seria, confidencial y profesional posible. Para que su hijo(a) pueda ser atendido(a) bajo esta modalidad de trabajo, es imprescindible que de forma previa tengamos su consentimiento informado, inequívoco y expreso en lo concerniente a la normativa de privacidad.

Por el presente documento, yo identificado con DNI $\mathrm{N}^{\circ}$ $y$ identificada con DNI $\mathrm{N}^{\circ} \ldots \ldots \ldots \ldots \ldots$, padres del(la) niño(a) autorizamos VOLUNTARIAMENTE que nuestro hijo(a) sea atendido(a) bajo la modalidad de teleasistencia psicológica en las áreas (conductual, psicológica, de aprendizaje y/o de lenguaje) que se requieran.

Así mismo, garantizamos tener conocimiento que el servicio de teleasistencia psicológica (evaluación e intervención) será brindado a través de la plataforma Microsoft Teams y correo electrónico, y que consistirá en lo siguiente:

1. Una entrevista con el padre y/o madre del niño(a) a través de videoconferencias y correo electrónico, donde se realizará el llenado de una ficha de datos de filiación, estrictamente necesarios para la evaluación e intervención.

2. Envío de vídeos del(la) niño(a) en situaciones cotidianas.

3. Observación del(la) niño(a) con y sin participación del especialista por medio de videoconferencias.

4. Llenado de fichas o listas de cotejo para la elaboración de un perfil detallado y diseño del plan de intervención.

5. Sesiones a través de videoconferencias con el(la) niño(a) y/o padres, destacándose el ROL ACTIVO de los padres.

6. Breve retroalimentación del desempeño del menor, después de cada sesión, así como un seguimiento durante la semana mediante el envío de un registro de conductas observadas por parte del padre y/o madre.

7. Comunicación vía online (videoconferencias y correo electrónico) tomando las medidas necesarias para proteger toda información personal.

Por tanto, firmamos en señal de conocimiento y aceptación.

Padre y/o apoderado

Nombre

DNI
Madre y/o apoderado

Nombre

DNI

\section{Ps. Alejandro Dioses Chocano \\ C.Ps.P. 1049 \\ Director de EOS Perú}

Lima, de de 2020 